\title{
Modelling the conversion between specific IgE test platforms for nut allergens in children and adolescents
}

\author{
Jennifer Hoang ${ }^{1}$, Alper Celik ${ }^{1}$, Christian Lupinek ${ }^{2}$, Rudolf Valenta ${ }^{2}$, Lucy Duan ${ }^{3}$, Ruixue \\ Dai $^{1}$, May Brydges ${ }^{1}$, Aimée Dubeau ${ }^{1}$, Claire Lépine ${ }^{1}$, Samantha Wong ${ }^{3}$, Mara \\ Alexanian-Farr ${ }^{3}$, Ahuva Magder ${ }^{3}$, Padmaja Subbarao ${ }^{3}$, Julia Upton ${ }^{3}$, Klara Schmidthaler ${ }^{2}$, \\ Zsolt Szepfalusi ${ }^{2}$, Arun Ramani ${ }^{3}$, and Thomas Eiwegger ${ }^{3}$ \\ ${ }^{1}$ Hospital for Sick Children Research Institute \\ ${ }^{2}$ Medical University of Vienna \\ ${ }^{3}$ Hospital for Sick Children
}

May 28, 2020

\begin{abstract}
Background: Multiplex tests allow for measurement of allergen-specific IgE responses to multiple allergen extracts and components and have several advantages for large cohort studies. Due to significant methodological differences, test systems are difficult to integrate in meta-analyses/systematic reviews since there is a lack of datasets with direct comparison. We aimed to create models for statistical integration of allergen-specific IgE to peanut/tree nut allergens from three IgE-test platforms. Methods: Plasma from Canadian and Austrian children with peanut/tree nut sensitization and a cohort of sensitized, high-risk, pre-school asthmatics (total $n=166$ ) were measured with three R\&D multiplex IgE test platforms: Allergy Explorer, ALEX (Macro Array Dx), MeDALL-chip (Mechanisms of Development of Allergy) (Thermo Fisher), and EUROLINE (EUROIMMUN). Skin prick test $(\mathrm{n}=51)$ and ImmunoCAP $(\mathrm{n}=62)$ results for extracts were available in a subset. Regression models (Multivariate Adaptive Regression Splines, local polynomial regression) were applied if $>30 \%$ of samples were positive to the allergen. Intra-test correlations between PR-10 and nsLTP allergens were assessed. Results: Using two regression methods, we demonstrated the ability to model allergen-specific relationships with acceptable measures of fit ( $2=94-56 \%$ ) for peanut and tree nut $\operatorname{sIgE}$ testing at the extract and component-level, in order from highest to lowest: Ara h 2, Ara h 6, Jug r 1, Ana o 3, Ara h 1, Jug r 2, Cor a 9. Conclusion: Our models support the notion that conversion is reasonably possible between sIgE multiplex platforms for allergen extracts and components and may provide options to aggregate data for future meta-analysis.
\end{abstract}

\section{Hosted file}

IgE Conversion_Main_v4.pdf available at https://authorea.com/users/327416/articles/454983modelling-the-conversion-between-specific-ige-test-platforms-for-nut-allergens-inchildren-and-adolescents 